\title{
Coarctação de aorta abdominal justarrenal associada à hipertensão renovascular e claudicação incapacitante: tratamento utilizando ponte ilíaco-birrenal e técnica "paving and cracking"
}

\author{
Coarctation of the abdominal aorta associated with renovascular hypertension and \\ incapacitating claudication: repair with iliac-birenal bypass and paving and cracking technique \\ Mateus Picada Corrêa ${ }^{1,2}$ (D), Francisco Costa Beber Lemanski (D), Jaber Nashat Saleh,2, Rafael Stevan Noel', \\ Renan Camargo Puton', Julio Cesar Bajerski ${ }^{1}$
}

\begin{abstract}
Resumo
Coarctação da aorta abdominal é uma causa rara de claudicação de membros inferiores e hipertensão refratária. O tratamento é complexo e exige conhecimento de diversas técnicas de reconstrução vascular. Apresentamos um caso de coarctação ao nível das artérias renais, seu tratamento e revisão da literatura. Paciente feminina, 65 anos, com hipertensão refratária desde os 35 anos, utilizando cinco medicações anti-hipertensivas em dose máxima. Pressão arterial média de 260/180mmHg e claudicação incapacitante (menos de 20 metros) bilateral. Angiotomografia computadorizada demonstrou coarctação de aorta justarrenal de $4 \mathrm{~mm}$ de maior diâmetro, calcificação circunferencial no local da estenose e tortuosidade da aorta infrarrenal. Foi submetida a tratamento híbrido, com ponte ilíaco-birrenal e implante de stent Advanta $\mathrm{V} 12$ no local da estenose. A paciente evoluiu satisfatoriamente e, 60 dias depois da cirurgia, apresentava-se com uma pressão arterial de 140/80 mm Hg, em uso de apenas duas medicações anti-hipertensivas e sem claudicação.
\end{abstract}

Palavras-chave: coarctação de aorta abdominal; ponte ilíaco-birrenal; hipertensão refratária; paving and cracking; endovascular.

\begin{abstract}
Coarctation of the abdominal aorta is a rare etiology of intermittent claudication and refractory hypertension. Treatment is complex and requires knowledge of several vascular reconstruction techniques. We report a case of aortic coarctation at the level of the renal arteries, describing its treatment and presenting a literature review. Female patient, 65 years old, with refractory hypertension since the age of 35 , using five antihypertensive medications at maximum doses. Blood pressure was $260 / 180 \mathrm{mmHg}$ and she had disabling claudication (less than 20 meters). Computed tomography angiography showed a $4 \mathrm{~mm}$ coarctation in the juxtarenal aorta, with circumferential calcification at the stenosis site, and tortuous infrarenal aorta. Hybrid repair was performed with an iliac-birenal bypass and implantation of an Advanta V12 stent at the stenosis site. The patient's postoperative course was satisfactory, she was free from claudication, and her blood pressure 60 days after surgery was 140/80 mmHg, taking two antihypertensive medications.
\end{abstract}

Keywords: abdominal aortic coarctation; iliac-birenal bypass; refractory hypertension; paving and cracking; endovascular.

Como citar: Corrêa MP, Lemanski FCB, Saleh JN, Noel RS, Puton RC, Bajerski JC. Coarctação de aorta abdominal justarrenal associada à hiper-tensão renovascular e claudicação incapacitante: tratamento utilizando ponte ilíacobirrenal e técnica "paving and cracking". J Vasc Bras. 2022;21:e20200175. https://doi.org/10.1590/1677-5449.200175 


\section{INTRODUÇÃO}

Coarctação da aorta é uma doença congênita rara, e a sua localização ao nível da aorta abdominal é uma variante ainda mais incomum da doença, representando $0,5-2 \%$ de todas as coarctações ${ }^{1,2}$. Quando está localizada ao nível da aorta abdominal, é uma causa de síndrome da aorta média (SAM) e geralmente está acompanhada de hipertensão renovascular e claudicação de membros inferiores.

A suspeita é feita com base na anamnese e no exame físico. A confirmação diagnóstica é feita com exames complementares, como a angiotomografia computadorizada (angio-TC). Diversas técnicas de intervenção cirúrgica foram descritas para o tratamento da $\mathrm{SAM}^{2}$. Neste artigo, relatamos o tratamento combinado de bypass ilíaco-birrenal com implante de stent por meio da técnica paving and cracking em uma paciente adulta com coarctação de aorta abdominal justarrenal.

O protocolo foi aprovado pelo Comitê de Ética de nossa instituição (parecer número 5.151.144).

\section{DESCRIÇÃO DO CASO}

Paciente do sexo feminino, 65 anos, procura atendimento em janeiro de $2015 \mathrm{com}$ queixa de claudicação intermitente bilateral há 2 anos, agora, para menos de 10 metros. Apresentava hipertensão arterial sistêmica (HAS) desde os 35 anos, refratária ao tratamento clínico, em uso de dose máxima de Metoprolol, Enalapril, Hidroclorotiazida, Anlodipino e Losartana, com crises hipertensivas de repetição. Refere história de acidente vascular encefálico hemorrágico há cinco anos, com remissão completa dos sintomas.

Ao exame físico, a pressão arterial (PA) era de 260/180 mmHg e demonstrava ausência total de pulsos bilateral nos membros inferiores. O exame neurológico revelou não ter sequelas.

A angio-TC de abdome demonstrou coarctação de aorta ao nível das artérias renais, medindo $4 \mathrm{~mm}$ de menor diâmetro (Figura 1A), com calcificação circunferencial no local da estenose e tortuosidade da aorta infrarrenal (Figura 2). Em virtude do tratamento cirúrgico de alto risco, a paciente optou por uma tentativa de tratamento clínico momentaneamente e, por piora da claudicação, optou por realizar procedimento três meses depois.

Através de um acesso transverso supraumbilical, foi realizada uma ponte da artéria ilíaca externa esquerda para a artéria renal esquerda e, desta, uma ponte em Y para a artéria renal direita, utilizando Dacron $6 \mathrm{~mm}$ com anastomose término-lateral (Figura 3). A prótese foi montada em bancada por uma segunda equipe durante a laparotomia. Após o término do bypass, foi realizada angioplastia da área da coarctação da aorta abdominal infrarrenal com implante de stent Advanta v12 10 x 38 mm (Getinge $\mathrm{AB}$, Suécia) e balão Powerflex Pro $12 \mathrm{~mm} \times 2 \mathrm{~cm}$ (Cordis, Santa Clara, EUA), tendo bom resultado angiográfico (Figura 1B). O procedimento teve duração de 5 horas, com sangramento estimado de 400 mL e sem necessidade de transfusão sanguínea. Foi iniciada profilaxia de tromboembolismo venoso (TEV) $24 \mathrm{~h}$ após o procedimento.

A paciente evoluiu satisfatoriamente, sem intercorrências no pós-operatório (PO), recebendo alta do Centro de Terapia Intensiva (CTI) no terceiro dia de PO e alta hospitalar no sétimo. No momento da última alta, apresentava PA de 160/140mmHg, estava em uso de três medicações anti-hipertensivas e possuía pulsos distais amplos.

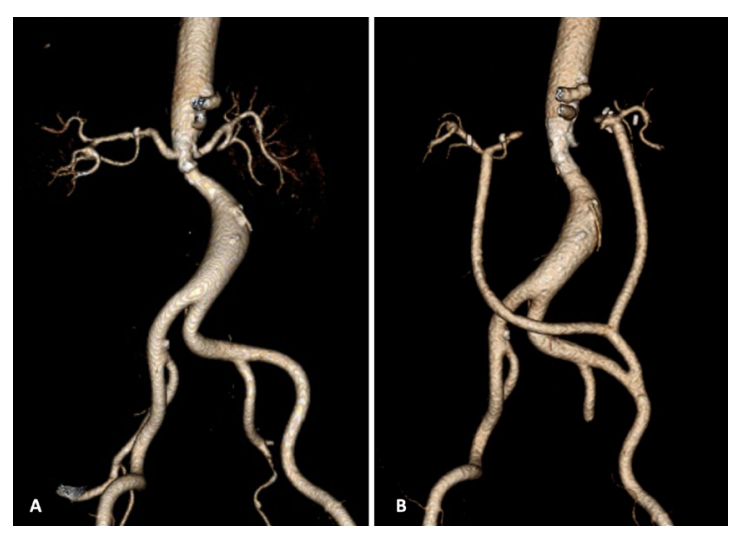

Figura 1. Reconstrução 3D de angiotomografia computadorizada de aorta abdominal. (A) Exame pré-operatório demonstrando redução do calibre da aorta ao nível das artérias renais, com calcificações parietais e discreta dilatação pós-estenótica; (B) Exame de controle pós-operatório demonstrando o aumento do diâmetro ao nível da estenose, com a ponte em Y para ambas as artérias renais, de origem da artéria ilíaca esquerda.
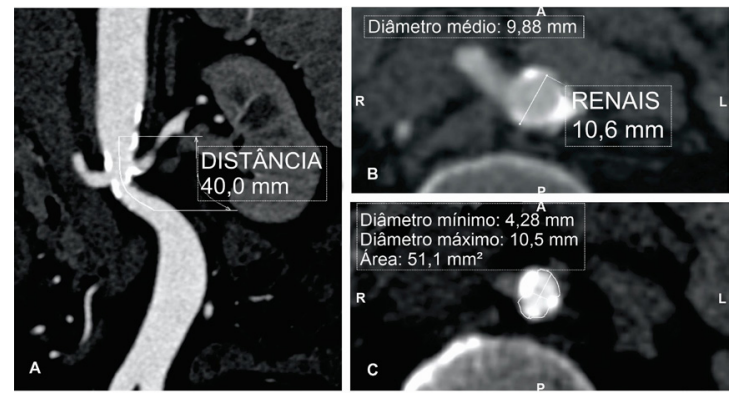

Figura 2. (A) Reconstrução em maximum intensity projection (MIP) da angiotomografia pré-operatória demonstrando um corte coronal ao nível das artérias renais, evidenciando as calcificações parietais ao nível das renais e o comprimento da estenose; (B) Cortes axiais demonstrando o diâmetro aórtico ao nível das artérias renais; (C) Diâmetro no ponto de maior estenose. 
A paciente permaneceu assintomática por um ano e está no quinto ano de acompanhamento. Anualmente, realizamos angioplastia do stent com balão $12 \times 40 \mathrm{~mm}$, sempre 30 dias depois da pressão da paciente voltar a subir. Não houve mais claudicação. A PA média da paciente é de $140 / 80 \mathrm{mmHg}$ com uso de apenas dois medicamentos anti-hipertensivos (Anlodipino e Losartana).

\section{DISCUSSÃO}

A coarctação de aorta é um defeito genético congênito que resulta no estreitamento da aorta. A coarctação pode afetar qualquer dos seus segmentos, porém é mais frequentemente encontrada na inserção do canal arterial, distal à emergência da artéria subclávia esquerda. Sendo uma anomalia infrequente, a coarctação de aorta corresponde de 4 a $6 \%$ de todas malformações cardíacas congênitas ${ }^{3}$. Seu diagnóstico geralmente é feito nos primeiros anos de vida, sendo a HAS o sinal alarmante, porém a alteração pode passar despercebida até a idade adulta, dependendo do grau da estenose.

A coarctação da aorta ao nível abdominal é uma doença rara, mais vista em crianças e adolescentes, que gera um estreitamento segmentar da artéria aorta abdominal, representando $2 \%$ de todas coarctações de aorta ${ }^{4}$. Faz parte da síndrome da aorta média, sendo uma de suas etiologias menos comuns ${ }^{1,2,4,5}$. Acredita-se ser decorrente da fusão incompleta das aortas dorsais na quarta semana de gestação. É uma doença congênita de etiologia desconhecida, podendo ter causa idiopática, genética (doença de Von Recklinghausen) ou adquirida (arterite de Takayasu e rubéola) ${ }^{1,2}$.
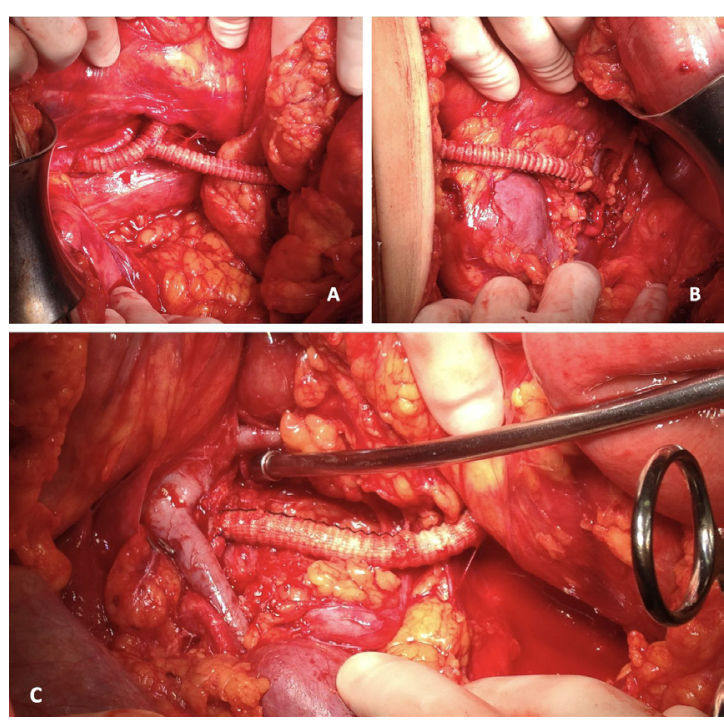

Figura 3. Imagens transoperatórias demonstrando a anastomose ilíaco-prótese ( $A$ - visão à esquerda), a anastomose da prótese com a artéria renal esquerda ( $B$ - visão à esquerda) e a anastomose da prótese com a artéria renal direita ( $C$ - visão à direita).
Os sintomas dependem da topografia da estenose, sendo os achados decorrentes da HAS os mais prevalentes. Coarctação justarrenal cursa com estenose das artérias renais, gerando estímulo à liberação da renina das células justaglomerulares do rim, causando hipertensão arterial secundária. Se a estenose não for significativa, a malformação pode passar despercebida até a idade adulta sem sintomas expressivos. Quando presentes, as manifestações clínicas estão relacionadas à hipertensão arterial grave, como cefaleia, epistaxe, insuficiência cardíaca ou dissecção aórtica. Além disso, na história clínica, pode haver claudicação dos membros inferiores devido à redução do fluxo sanguíneo. $\mathrm{O}$ uso da angio-TC para diagnóstico e avaliação pré-operatória está bemestabelecido, fornecendo informações importantes acerca da anatomia arterial da região acometida ${ }^{6,7}$.

O tratamento cirúrgico é indicado para o controle da HAS refratária ao tratamento clínico e para melhora da claudicação ${ }^{2,7}$. A técnica operatória deve ser individualizada, levando em consideração a anatomia da lesão, o envolvimento de artérias viscerais e o risco cardiovascular do paciente ${ }^{6}$. Diversas técnicas podem ser utilizadas para a reconstrução renovascular e da aorta: bypass aorto-aórtico, aortoplastia com retalho, angioplastia endovascular, reconstrução de artéria renal e de artérias viscerais ${ }^{2,6-8}$. Na paciente relatada, foi optado pelo bypass ilíaco-birrenal para reconstrução da vascularização renal e, em seguida, a implatação do stent por "paving and cracking" para reparo da coartação da aorta abdominal (Figura 4). Existem apenas 17 casos mencionados na literatura mundial sobre a ocorrência de coarctação de aorta abdominal em adultos com idade superior a 20 anos, visto que a maior parte dos diagnósticos ocorrem na infância, e nenhum na literatura brasileira ${ }^{6,9-17}$.

Dada a anatomia da lesão, algumas considerações a respeito do planejamento do procedimento devem ser destacadas. Primeiro, não foi optada pela reconstrução

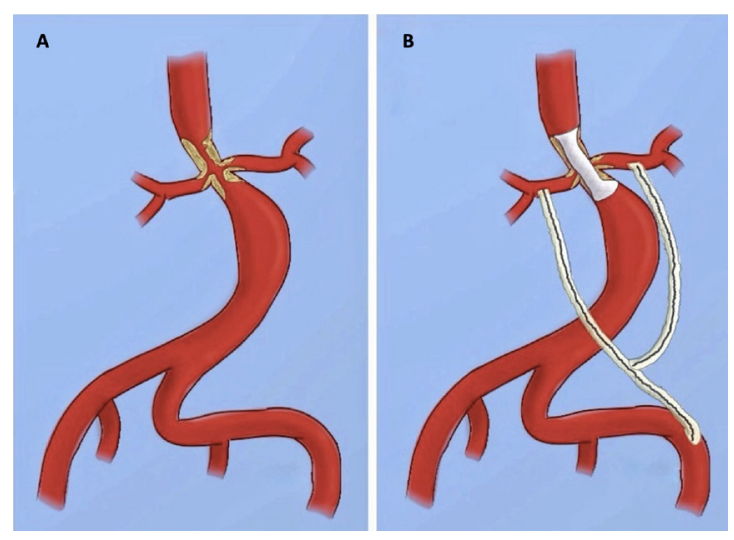

Figura 4. Representação esquemática do procedimento cirúrgico no pré- (A) e pós-operatório (B). 
in situ da aorta com reanastomose das artérias renais para se evitar o clampeamento suprarrenal e a isquemia renal bilateral simultânea, o que poderia aumentar a morbidade pós-operatória com risco de insuficiência renal aguda ${ }^{18,19}$. Realizando uma ponte ilíaco-renal bilateral com anastomose término-lateral, não tivemos isquemia renal prolongada. A conformação do bypass foi extrapolada da recomendada por Oderich et al. para as reconstruções retrógradas ilíaco-mesentérica superior, que aumentam a perviedade em longo prazo por reduzir o risco de turbilhonamento ${ }^{20}$.

A opção de realizar um acesso transverso em vez de longitudinal foi sugerida pela equipe de Urologia, a fim de facilitar o acesso aos hilos renais, o que se confirmou conveniente no transoperatório. Com o amplo acesso, foi possível acessar desde a bifurcação da artéria ilíaca comum até as artérias renais, nosso alvo.

O stent v12 10x38 foi escolhido por ser, na ocasião, o stent revestido de maior diâmetro disponível, tendo a opção de aumentar o diâmetro para $12 \mathrm{~mm}$ com um balão não complacente (Figura 5). A decisão de se utilizar stent revestido reduziu o tempo cirúrgico por não haver necessidade de acesso aos óstios das artérias renais, criando uma "ligadura endovascular" proximal. Além disto, stents revestidos são indicados na técnica de "paving and cracking" 21.

Essa técnica foi desenvolvida para facilitar o implante de endopróteses aórticas através de artérias ilíacas severamente calcificadas. Após o acesso às artérias femorais comuns e a angioplastia da artéria ilíaca, é realizado o implante de uma endoprótese ou stent revestido de diâmetro compatível com a artéria, no intuito de realinhar o lúmen arterial (paving). Sequencialmente, realiza-se uma sobredilatação da artéria com balão de diâmetro de $10 \mathrm{~mm}$ em toda a área do stent, fragmentando a placa (cracking). $\mathrm{O}$ tecido do stent previne embolização distal ou sangramento. Uma vez que a artéria ilíaca tenha sido revestida e dilatada,
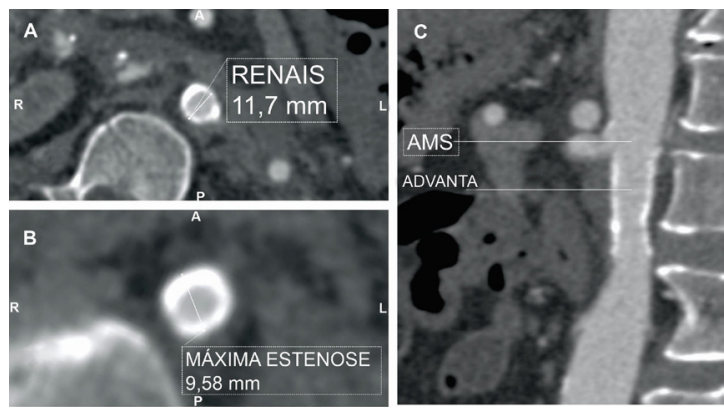

Figura 5. Reconstrução em maximum intensity projection (MIP) da angiotomografia pós-operatória de controle demonstrando os mesmos cortes da Figura 2: um corte coronal ao nível das artérias renais, evidenciando o aspecto do implante do stent (A), e cortes axiais demonstrando o diâmetro aórtico ao nível das artérias renais (B) e o diâmetro no ponto de maior estenose (C). AMS = artéria mesentérica superior. a endoprótese aórtica pode ser inserida facilmente e implantada de maneira padrão ${ }^{21}$. No caso em questão, realizamos uma sobredilatação com balão $12 \mathrm{~mm}$, e o stent revestido impediu sangramento, caso tenha ocorrido eventual ruptura da parede arterial.

\section{CONCLUSÃO}

A coarctação da aorta abdominal é uma etiologia rara de claudicação simétrica de membros inferiores ou hipertensão refratária; todavia, na associação desses sintomas, o diagnóstico deve sempre ser considerado. $\mathrm{O}$ tratamento é complexo e exige conhecimento de diversas técnicas de reconstrução vascular para o seu sucesso, tendo como objetivo reverter a HAS e a claudicação do paciente.

\section{REFERÊNCIAS}

1. Connolly JE, Wilson SE, Lawrence PL, Fujitani RM. Middle aortic syndrome: distal thoracic and abdominal coarctation, a disorder with multiple etiologies. J Am Coll Surg. 2002;194(6):774-81. http:// dx.doi.org/10.1016/S1072-7515(02)01144-4. PMid:12081068.

2. Rumman RK, Nickel C, Matsuda-Abedini M, et al. Disease beyond the arch: a systematic review of middle aortic syndrome in childhood. Am J Hypertens. 2015;28(7):833-46. http://dx.doi. org/10.1093/ajh/hpu296. PMid:25631383.

3. Reller MD, Strickland MJ, Riehle-Colarusso T, Mahle WT, Correa A. Prevalence of congenital heart defects in metropolitan Atlanta, 1998-2005. J Pediatr. 2008;153(6):807-13. http://dx.doi.org/10.1016/j. jpeds.2008.05.059. PMid:18657826.

4. Cohen JR, Birnbaum E. Coarctation of the abdominal aorta. J Vasc Surg. 1988;8(2):160-4. http://dx.doi.org/10.1016/07415214(88)90404-1. PMid:3398174.

5. Sen PK, Kinare SG, Engineer SD, Parulkar GB. The middle aortic syndrome. Br Heart J. 1963;25(5):610-8. http://dx.doi.org/10.1136/ hrt.25.5.610. PMid:14063008.

6. Stanley JC, Criado E, Eliason JL, Upchurch GR Jr, Berguer R, Rectenwald JE. Abdominal aortic coarctation: surgical treatment of 53 patients with a thoracoabdominal bypass, patch aortoplasty, or interposition aortoaortic graft. J Vasc Surg. 2008;48(5):1073-82. http://dx.doi.org/10.1016/j.jvs.2008.05.078. PMid:18692352.

7. Delis KT, Gloviczki P. Middle aortic syndrome: from presentation to contemporary open surgical and endovascular treatment. Perspect Vasc Surg Endovasc Ther. 2005;17(3):187-203. http:// dx.doi.org/10.1177/153100350501700302. PMid:16273154.

8. Sandmann W, Dueppers P, Pourhassan S, Voiculescu A, Klee D, Balzer KM. Early and long-term results after reconstructive surgery in 42 children and two young adults with renovascular hypertension due to fibromuscular dysplasia and middle aortic syndrome. Eur J Vasc Endovasc Surg. 2014;47(5):509-16. http:// dx.doi.org/10.1016/j.ejvs.2013.12.012. PMid:24583096.

9. Ghazi P, Haji-Zeinali AM, Ghasemi M, Pour MZ. Transcatheter stent implantation for the treatment of abdominal aortic coarctation and right renal artery stenosis in takayasu's arteritis: a case with a 4-year follow up. Hellenic J Cardiol. 2011;52(2):182-5. PMid:21478132.

10. Savlania A, Parameshwarappa SK, Viswanathan S, Raman KT, Madathipat U. Extra-anatomic neo-aorta for adult abdominal aortic coarctation. ANZ J Surg. 2014;84(12):988-90. http://dx.doi. org/10.1111/ans.12358. PMid:24172236. 
11. Moresco KP, Shapiro RS. Abdominal aortic coarctation: CT, MRI, and angiographic correlation. Comput Med Imaging Graph. 1995;19(5):427-30. http://dx.doi.org/10.1016/0895-6111(95)000372. PMid:8734781.

12. Iwata A, Takahashi Y, Ohgi K, Sakuta M. A case of spinal hemorrhage associated with abdominal aortic coarctation. Rinsho Shinkeigaku. 1997;37(5):413-6. PMid:9294331.

13. Mallios A, Boura B, Combes M, Blebea J. Abdominal aortic coarctation in a middle aged adult. J Vasc Surg. 2015;61(1):240. http://dx.doi.org/10.1016/j.jvs.2013.09.015. PMid:25534979.

14. Koksal C, Demirci S, Koksal GM, Zengin M. An infrarenal abdominal aortic coarctation. Surg Radiol Anat. 2005;27(1):71-3. http://dx.doi. org/10.1007/s00276-004-0288-1. PMid:15517260.

15. Nawa S, Ikeda E, Kubo M, et al. Multisaccular aneurysm developing in association with abdominal aortic coarctation: report of a surgical case. Ann Thorac Cardiovasc Surg. 2003;9(5):326-9. PMid:14672531.

16. Paroni R, Astuni $M$, Baroni $C$, et al. Abdominal aortic coarctation inducing aortic occlusion and renovascular hypertension. Cardiovasc Surg. 1991;32(6):770-4. PMid:1752897.

17. Graham LM, Zelenock GB, Erlandson EE, Coran AG, Lindenauer SM, Stanley JC. Abdominal aortic coarctation and segmental hypoplasia. Surgery. 1979;86(4):519-29. PMid:483161.

18. Breckwoldt WL, Mackey WC, Belkin M, O'Donnell TFJr. The effect of suprarenal cross-clamping on abdominal aortic aneurysm repair. Arch Surg. 1992;127(5):520-4. http://dx.doi.org/10.1001/ archsurg.1992.01420050040004. PMid:1575621.

19. Yokoyama N, Nonaka T, Kimura N, et al. Acute kidney injury following elective open aortic repair with suprarenal clamping. Ann Vasc Dis. 2020;13(1):45-51. http://dx.doi.org/10.3400/avd. oa.19-00095. PMid:32273921.

20. Oderich GS, Gloviczki P, Bower TC. Open surgical treatment for chronic mesenteric ischemia in the endovascular era: when it is necessary and what is the preferred technique? Semin Vasc Surg. 2010;23(1):36-46. http://dx.doi.org/10.1053/j.semvascsurg.2009.12.005. PMid:20298948.

21. Hinchliffe RJ, Ivancev K, Sonesson B, Malina M. “Paving and cracking”: an endovascular technique to facilitate the introduction of aortic stent-grafts through stenosed iliac arteries. J Endovasc Ther. 2007;14(5):630-3. http://dx.doi.org/10.1177/152660280701400505. PMid:17924727.

Correspondência Mateus Picada Corrêa Instituto Vascular de Passo Fundo - INVASC Rua Capitão Araújo, 297, $12^{\circ}$ andar CEP 99010-200 - Passo Fundo (RS), Brasil Tel.: (54) 3045-3340 E-mail:drmateus@invascrs.org

Informações sobre os autores MPC - Professor de Cirurgia Vascular, Universidade de Passo Fundo

(UPF); Professor de Cirurgia Vascular, IMED; Cirurgião Vascular, Endovascular e Radiologista Intervencionista, Instituto Vascular de Passo Fundo (Invasc).

FCBL - Acadêmico de Medicina, Universidade de Passo Fundo (UPF).

JNS - Professor de Cirurgia Vascular e Anatomia, Universidade de Passo Fundo (UPF); Cirurgião Vascular, Endovascular e Radiologista Intervencionista, Instituto Vascular de Passo Fundo (Invasc). RSN - Cirurgião Vascular e Endovascular, Instituto Vascular de Passo Fundo (Invasc).

RCP - Cirurgião Vascular, Instituto Vascular de Passo Fundo (Invasc). JCB - Cirurgião Vascular, Endovascular e Radiologista Intervencionista, Instituto Vascular de Passo Fundo (Invasc).

Contribuições dos autores Concepção e desenho do estudo: MPC Análise e interpretação dos dados: MPC, FCBL Coleta de dados: MPC, FCBL Redação do artigo: MPC, FCBL Revisão crítica do texto: MPC, JNS, RSN, JCB, RCP Aprovação final do artigo*: MPC, JNS, RSN, JCB, RCP Análise estatística: N/A. Responsabilidade geral pelo estudo: MPC

*Todos os autores leram e aprovaram a versão final submetida ao J Vasc Bras. 